\title{
A novel cannulation technique for difficult urethral catheterization
}

\author{
Mehmet Kaynar, Murat Akand, Serdar Goktas \\ Department of Urology, Selcuk University, Faculty of Medicine, Konya, Turkey.
}

\begin{abstract}
Summary Introduction: To propose a novel cannulation technique for difficult urethral catheterization procedures.

Technique: The sheath tip of an intravenous catheter is cut off, replaced to the needle tip and pushed through the distal drainage side hole to Foley catheter tip, and finally withdrawn for cannulation. In situations making urethral catheterization difficult, a guide wire is placed under direct vision. The modified Foley catheter is slid successfully over the guide wire from its distal end throughout the urethral passage into the bladder.

Results: The modified Foley catheter was used successfully in our clinic in cases requiring difficult urethral catheterization. Conclusions: This easy and rapid modification of a Foley catheter may minimize the potential complications of blind catheter placement in standard catheterization.
\end{abstract}

KEY WORDS: Technique; Difficult; Urethral catheterization.

Submitted 5 October 2015; Accepted 11 November 2015

\section{INTRODUCTION}

Despite the developments in minimally invasive techniques and invention of novel endourological instruments, difficulties and failures in urethral catheterization may be unavoidable especially in male patients. Urologist may have difficulties in urethral catheterization caused by urethral strictures, iatrogenic false passages, large obstructive prostates, and especially in the presence of a large median lobe that may associated to undermining of the bladder trigone after an excessive transurethral resection of the bladder neck. Several techniques and instrument have been described in the literature in case of misdirection or misplacement of an indwelling urethral catheter (1-3). The present manuscript is about a novel, alternative, simple, and safe cannulation technique to be used in difficult urethral catheterization.

\section{TECHNIQUE AND MATERIAL}

A 16-gauge intravenous (IV) catheter, a 0.038 inch PTFE-Nitinol guidewire with hydrophilic floppy tip (SensorTM Boston Scientific Corporation) or any other teflon coated spiral wound guide wire, PC guide wire and double J guide wire, an indwelling $22 \mathrm{~F}$ three-way
Foley catheter (Rüsch Gold TMGermany), and a scalpel are the necessary tools (Figure 1A). The tip of the IV catheter sheath is cut off up to $4 \mathrm{~mm}$ and replaced to the needle tip (Figures 1B, 1C). The IV catheter with the cut and replaced sheath tip is pushed through the distal drainage side hole of the urethral catheter to the center of the Foley catheter tip. The intravenous catheter needle and proximal sheath are withdrawn (Figure 2A). Upon withdrawal, the $4 \mathrm{~mm}$ cut sheath remains spontaneously in the Foley catheter tip. Yet, the length of the tip of the sheath cannula sticking out of the Foley catheter may be $1 \mathrm{~mm}$ or less. In the present manuscripts it is longer in order to depict the technique (Figure 2B). Depending on the situation present, the guide wire is fed past the undermining bladder trigone and placed into the bladder under direct vision using a resectoscope. After the placement of the guide wire, the modified Foley catheter with the $4 \mathrm{~mm}$ sheath tip for cannulation is slid successfully over the guide wire from its distal end throughout the urethral passage into the bladder (Figure 2C). Finally, the balloon is filled and the guide wire is removed.

The modified Foley catheter with the sheath tip for cannulation was used successfully in our clinic in cases requiring difficult urethral catheterization such as after internal urethrotomy in urethral strictures, flexible cystoscopy and cystourethroscopy in false passages, and transurethral resection of the prostate with emergent undermining of bladder trigone.

\section{Discussion}

Urologist develop their own ways with time and experience to dwell a catheter in challenging situations using different tools and methods as there is not a standard procedure. The present manuscript describes the use of a Foley catheter minimizing urethral trauma and that can be placed easily and safely into the bladder using a guide wire. The use short rigid ureteroscope placed into Foley catheter in urethral catheterization may not be suitable with small size Foley catheters and in case of hemorrhagy disturbing the view of the urethral passage (3). Peel-away sheath may not be a routine tool as in extreme urethral strictures hindering the placement of cystoscopy or resectoscope (2). Blitz has defined a similar technique using an IV catheter placed into a Foley catheter to enable easy passage of hydrophilic guide wire. However, in non- 


\section{Figure 1.}

A: 16-gauge intravenous (IV) catheter, 0.038 in PTFENitinolGuidewire with Hydrophilic floppy Tip, Indwelling 22-F 3 way Foley catheters, and bistoury.

B: The tip of the IV catheter sheath is cut off up to $4 \mathrm{~mm}$.

C: Replaced sheath to the needle tip.

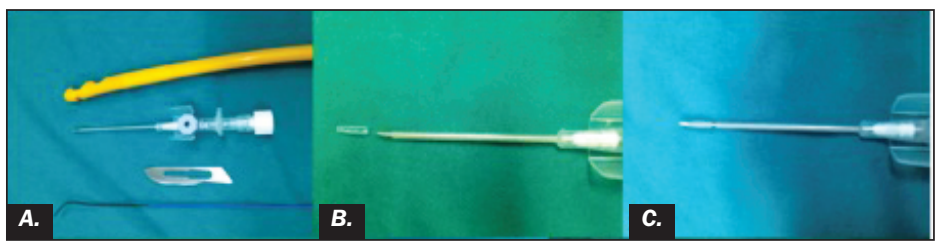

\section{Figure 2.}

A: The IV catheter with the cut and replaced sheath tip is pushed through the distal drainage side hole of the urethral catheter to the center of the Foley catheter tip.

B: The intravenous catheter needle and proximal sheath is withdrawn. Sheath remains spontaneously in the Foley catheter tip.

C: The Foley catheter with the sheath tip is slid over the guide wire into the bladder.

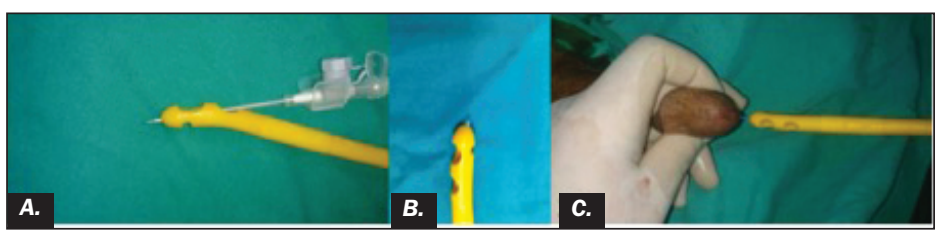

hydrophilic guide wires, the friction between the guide wire and Foley catheter makes the advancement of the catheter difficult. Cutting the catheter tip on the other

\section{REFERENCES}

1. Blitz BF. A Simple Method Using Hydrophilic Guide Wires for the Difficult Urethral Catheterization. Urology. 1995; 46:99-100.

2. Lowe MA, Defalco AJ. Endourologic Technique for Catheter Placement after TURP, Prostatectomy, and

hand, makes the Foley catheter more disposed to deformation of the catheter tip and balloon as well as snagging, during urethral passage (1). Yet, our modified Foley catheter enables permanent cannulation with all type of guide wires minimizing guide wire buckling and Foley catheter snagging due to the lack of friction in impacted conditions; since the catheter tip is not deformed, gait will become less resistant to advancement enabling into the bladder.

The proposed technique can be used successfully with all type of guide wires and catheters varying in size and type. It is rapidly and easily applied with common urological tools and it minimizes the potential complications of blind catheter placement in standard catheterization.

\section{ACKNOWLEDGMENT}

We would like to express our gratitude to the medical assistant of the urology operation theatre Mr. Ennur Civelek for his contribution. Difficult Urethroscopy. Urology. 1992; 40:461-63.

3. Rozanski TA, Salazar F, Thompson IM. Direct Vision Bladder Catheterization Using a Short Rigid Ureteroscope. Urology. 1998; 51:827-28.

\section{Correspondence}

Mehmet Kaynar, MD (Corresponding Author)

mekaynar@gmail.com

Selçuk Üniversitesi Alaeddin Keykubat Kampüsü

42075 Selçuklu-Konya, Turkey

Murat Akand, MD

Serdar Goktas, MD

Department of Urology, Selcuk University, Faculty of Medicine

Konya, Turkey 